\title{
Using amino acids co-occurrence matrices and explainability model to investigate patterns in dengue virus proteins
}

\author{
Leonardo R. Souza ${ }^{1 *}$, Juan G. Colonna', Joseana M. Comodaro ${ }^{2}$ and Felipe G. Naveca ${ }^{3}$
}

\author{
${ }^{*}$ Correspondence: \\ rdsouza.leonardo@icomp. \\ ufam.edu.br \\ ${ }^{1}$ Institute of Computing, \\ Federal University \\ of Amazonas, General \\ Rodrigo Octavio Avenue, \\ Manaus, Amazonas, Brazil \\ Full list of author information \\ is available at the end of the \\ article
}

\begin{abstract}
Background: Dengue is a common vector-borne disease in tropical countries caused by the Dengue virus. This virus may trigger a disease with several symptoms like fever, headache, nausea, vomiting, and muscle pain. Indeed, dengue illness may also present more severe and life-threatening conditions like hemorrhagic fever and dengue shock syndrome. The causes that lead hosts to develop severe infections are multifactorial and not fully understood. However, it is hypothesized that different viral genome signatures may partially contribute to the disease outcome. Therefore, it is plausible to suggest that deeper DENV genetic information analysis may bring new clues about genetic markers linked to severe illness.
\end{abstract}

Method: Pattern recognition in very long protein sequences is a challenge. To overcome this difficulty, we map protein chains onto matrix data structures that reveal patterns and allow us to classify dengue proteins associated with severe illness outcomes in human hosts. Our analysis uses co-occurrence of amino acids to build the matrices and Random Forests to classify them. We then interpret the classification model using SHAP Values to identify which amino acid co-occurrences increase the likelihood of severe outcomes.

Results: We trained ten binary classifiers, one for each dengue virus protein sequence. We assessed the classifier performance through five metrics: PR-AUC, ROC-AUC, F1-score, Precision and Recall. The highest score on all metrics corresponds to the protein $\mathrm{E}$ with a 95\% confidence interval. We also compared the means of the classification metrics using the Tukey HSD statistical test. In four of five metrics, protein $\mathrm{E}$ was statistically different from proteins M, NS1, NS2A, NS2B, NS3, NS4A, NS4B and NS5, showing that $\mathrm{E}$ markers has a greater chance to be associated with severe dengue. Furthermore, the amino acid co-occurrence matrix highlight pairs of amino acids within Domain 1 of E protein that may be associated with the classification result.

Conclusion: We show the co-occurrence patterns of amino acids present in the protein sequences that most correlate with severe dengue. This evidence, used by the classification model and verified by statistical tests, mainly associates the E protein with the severe outcome of dengue in human hosts. In addition, we present information suggesting that patterns associated with such severe cases can be found mostly in Domain 1, inside protein E. Altogether, our results may aid in developing new author(s) and the source, provide a link to the Creative Commons licence, and indicate if changes were made. The images or other third party material in this article are included in the article's Creative Commons licence, unless indicated otherwise in a credit line to the material. If material is not included in the article's Creative Commons licence and your intended use is not permitted by statutory regulation or exceeds the permitted use, you will need to obtain permission directly from the copyright holder. To view a copy of this licence, visit http:// creativecommons.org/licenses/by/4.0/. The Creative Commons Public Domain Dedication waiver (http://creativecommons.org/publi cdomain/zero/1.0/) applies to the data made available in this article, unless otherwise stated in a credit line to the data. 
treatments and being the target of debate on new theories regarding the infection caused by dengue in human hosts.

Keywords: Dengue virus, Protein amino acid co-occurrence, Machine learning

\section{Background}

Dengue is a viral infection that in most cases leads to a febrile syndrome without high clinical risk, accompanied by headaches, orbital, muscles and joints aches, nausea, vomiting and skin rashes. However, cases of severe dengue, like the dengue shock syndrome, occur with a certain frequency. Patients with severe dengue conditions may have difficulty breathing, severe bleeding, severe abdominal pain, frequent vomiting, fluid retention and fatigue. This combination of symptoms makes severe dengue potentially fatal [1]. Early identification of the infection combined with appropriate treatments can reduce the chances of fatality by more than $99 \%[2,3]$.

Dengue cases may be found in all continents, excluding Antarctica. However, the virus has established and persisted endemically in urban areas of tropical and subtropical, which are favorable for the maintenance of the Aedes aegypti mosquito, the main vector of dengue $[4,5]$. Statistical studies indicate that approximately 390 million people are infected every year, of which 96 million need some kind of medical attention [6]. Analyzes of infected patient samples indicate cases of fatal infection between $2.5 \%$ and $5.9 \%[7,8]$. Although the global distribution of dengue is uncertain, research indicates the establishment of the Aedes aegypti mosquito in 129 countries, suggesting a population of 3.9 billion people at risk for the infection $[6,9,10]$.

Dengue viruses are divided into four groups of antigenically distinct serotypes, this feature enables dengue reinfections through new serotypes for the host's immune system [11]. Despite this, it is believed that primary dengue infection generates heterotypic immunity within 1-3 years, and that secondary infection causes extensive cross-protection, resulting in rare post-secondary infections [12-14]. However, secondary infection increases the risk of severe dengue [15].

The genetic material of the virus consists of single-stranded RNA (single-stranded RNA-ssRNA) with approximately 10,200 nucleotides and can be represented by a sequence of characters taken from a specific alphabet. To preserve the biological functions of each protein, specific relationships between nucleotides occur in every coding RNA. Thus, the dengue RNA coding region is divided into three structural proteins that make up the virion: $C, M$ and $E$ and; in seven non-structural proteins used in viral replication: NS1, NS2A, NS2B, NS3, NS4A, NS4B and NS5. In the complete genome illustrated in Fig. 1, the regulatory regions 5'UTR and 3'UTR that do not translate proteins, called ncRNA $[16,17]$ are also observable.

Each protein is responsible for a specific task. Protein E is responsible for recognition and entry into the cell to be infected [18], while protein NS1 participates of RNA replication and helps in the formation of immune complexes [19, 20]. The NS2A protein is important for viral pathogenesis, while the NS2B and NS3 proteins play an important role in viral protease functions [21-23]. The NS4A protein is associated with the $\mathrm{M}$ protein through internal regions and performs membrane rearrangement [24]. There is evidence that NS4A and NS4B can function cooperatively in viral replication and anti-host response [24, 25]. Finally, the NS5 protein bypasses the infected 


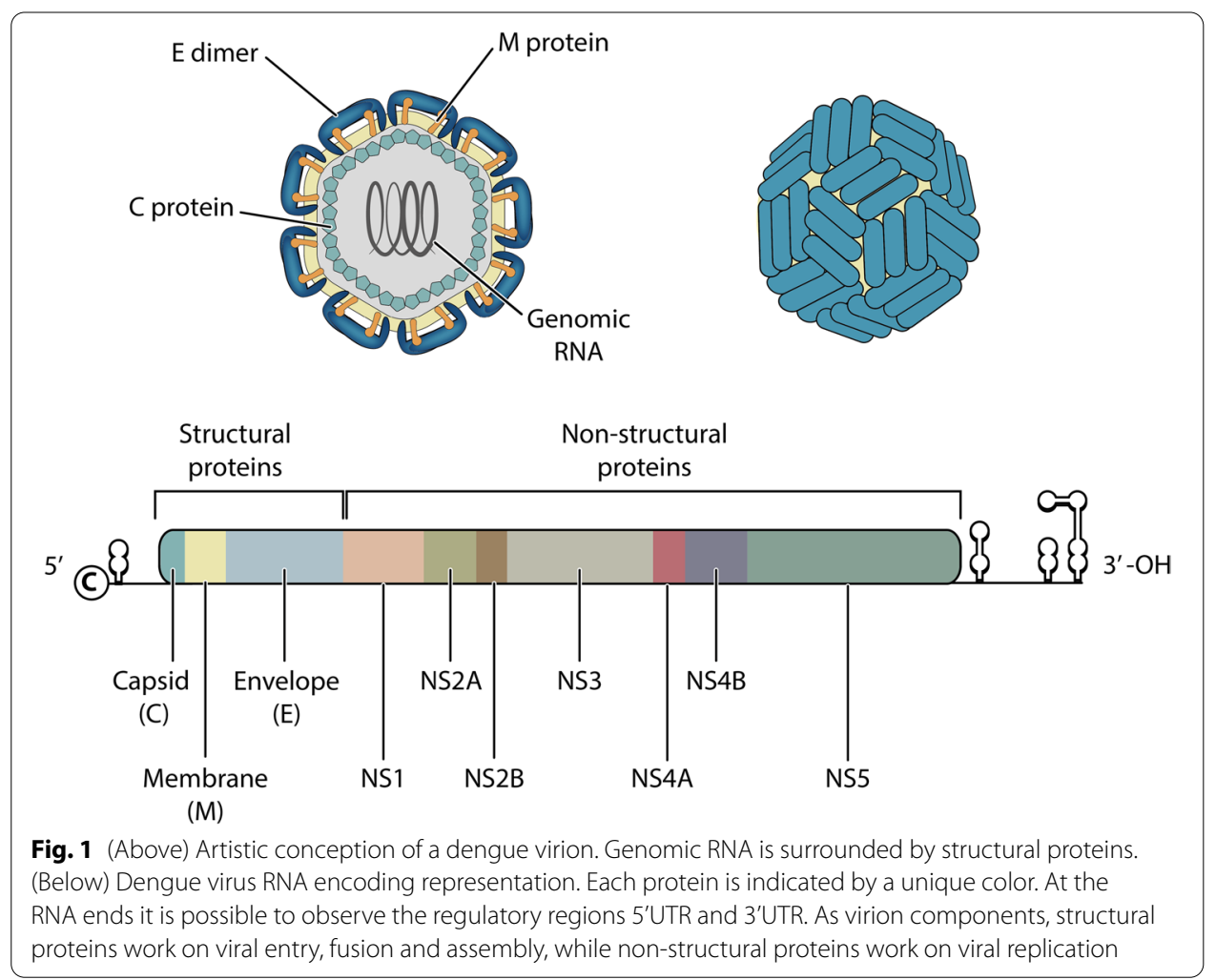

organism's innate immune response system and is the viral RNA-dependent RNA polymerase [26, 27].

In this study, we explored and compared all these dengue proteins looking for amino acid patterns that may be associated with severe dengue. Machine learning algorithms rely on numerical inputs to perform prediction tasks. Based on this need, we propose the encoding of protein coding sequence in co-occurrence matrices of amino acids.

For this, we assembled a data set, in which the coding RNA sequences were aligned, translated and segmented to obtain the deduced proteins. We then encode these proteins into amino acid co-occurrence matrices, labeling them with the associated degrees of infection. Subsequently, these matrices are classified by a Random Forest (RF). Finally, the instance-label associations learned by the classifier are interpreted locally using SHAP Values (SHapley Additive exPlanations), revealing the co-occurrence patterns of amino acids that increase the probability of severe dengue in the sample.

Our results suggest that protein $\mathrm{E}$ has a better association with the degree of infection, with more relevant patterns for severity present in the region called Domain 1 of this protein. In addition to these results, the database of this work can be considered an additional contribution, as we provide data from protein-segmented dengue RNA samples containing information on the serotype and severity of the host-associated infection.

\section{Methods}

\section{Framework for severe dengue explanation}

The general objective of this research is to explore, through a machine learning (ML) explainability technique, the interaction between amino acids present in dengue proteins 
and how they generate patterns capable of associated the severity of dengue infection. For this, our framework is divided into 5 steps, namely: (1) viral RNA alignment and protein segmentation so that they can be explored independently; (2) sequence normalization and tokenization as steps to standardize and obtain protein amino acids; (3) generation of co-occurrence matrices of amino acids that will serve as training data for the classifier; (4) prediction of the degree of infection through the Random Forest (RF) algorithm and; (5) local explanation of the RF classification model for the training samples in order to extract sets of co-occurrences of significant amino acids for prediction of severe dengue.

\section{Input data}

Proteins are chains of amino acids, such that amino acids are represented by characters taken from a specific alphabet known as IUPAC (International Union of Pure and Applied Chemistry) [28]. Let $P$ be a protein such that, for any $p_{i} \in A, P$ can be mathematically represented by the series $P=p_{1} p_{2} p_{3} \ldots p_{n-1} p_{n}$, where $p_{i}$ is a amino acids, $A$ is the alphabet and $n$ is the number of amino acids in the protein.

\section{Data scraping}

Despite the large amount of dengue genomes publicly available for research in gene sequence repositories, we found a great scarcity of samples labeled with the clinical picture of the infected patient. Therefore, we mine the NCBI (National Center for Biotechnology Information) and NCBI Virus Variation repositories in search of dengue genomic sequences labeled with the patient's clinical outcome. A total of 562 labeled samples were obtained. Of this total, 61 samples have the complete dengue genome encoding all 10 proteins. For each protein, we generate a separate data file in the following order: Additional file 1: $\mathrm{C}$ protein, Additional file 2: $\mathrm{M}$ protein, Additional file 3: $\mathrm{E}$ protein, Additional file 4: NS1 protein, Additional file 5: NS2A protein, Additional file 6: NS2B protein, Additional file 7: NS3 protein, Additional file 8: NS4A protein, Additional file 9: NS4B protein, and Additional file 10: NS5 protein. This subset of carefully selected sequences is another a contribution of our work. We also make a copy available in a public repository via the link https://doi.org/10.5281/zenodo.5885637.

The labels found were: dengue fever (DF), dengue hemorrhagic fever (DHF) and dengue shock syndrome (DSS). Given the low amount of DHF and DSS samples and because they are severe cases of dengue, we performed the binary labeling of our database, where DF became "classic dengue" and DHF and DSS, "severe dengue". All samples, with the exception of two samples collected from the spleen, were collected through blood material isolated from infected humans between 1985 and 2017. Data are from Brazil, Cambodia, Chile, China, Colombia, Cuba, Spain, Philippines, Ghana, India, Indonesia, Japan, Malaysia, Mexico, Paraguay, French Polynesia, Sri Lanka, Vietnam, Thailand and Taiwan (Republic of China).

\section{Protein sequences pre-processing}

To avoid non-conformities in the classification and explanation of results steps, the protein sequences go through the steps of: alignment, normalization and tokenization, as illustrated in Fig. 2. 


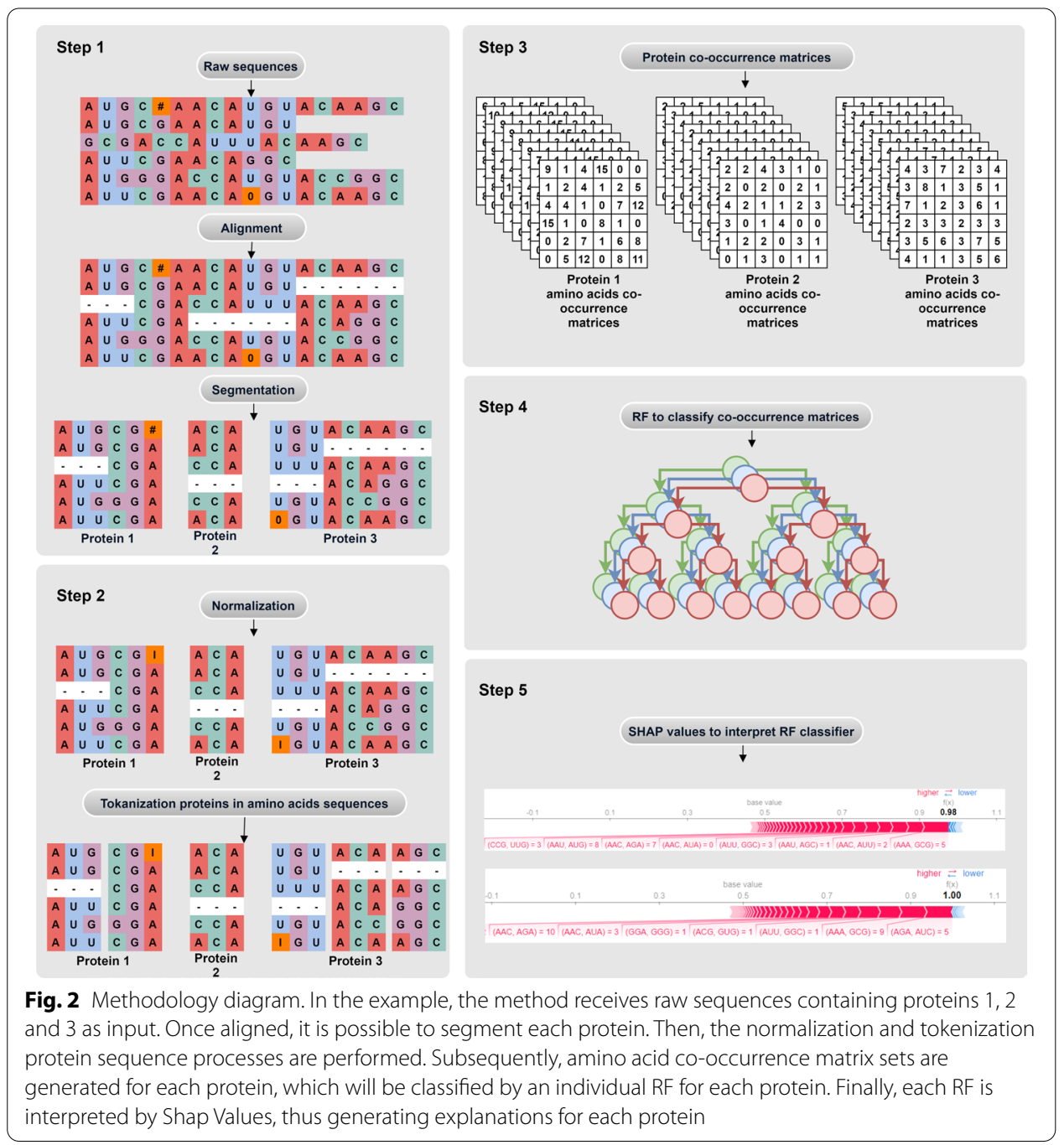

\section{Sequence alignment and segmentation}

The sequences were aligned using the MUSCLE algorithm available in the UGENE [29] software. MUSCLE is a three-stage alignment algorithm for multiple sequences [30]. After the alignment is completed, protein segmentation is performed. The segmentation of enconding sequences into deduced proteins was performed based on the reference sequences available in GenBank for each dengue virus serotype.

Sequence alignment allows for standardization of raw data samples, filling incomplete sequences with gaps so that they line up with 61 samples with complete genomes, allowing the creation of a database for each protein (Fig. 2). The sequence alignment process is based on the calculation of similarity of conserved regions between sequences. Therefore, it is natural that the alignment adds gaps in partially incomplete sequences so that the conserved regions of each sequence are aligned, increasing the similarity between sequences [30-32]. This procedure can result in extensive gap regions for very incomplete sequences, causing entire proteins to be represented solely by gaps. To get around this problem, before any processing to generate co-occurrence matrices, we chose to remove samples formed by more than $15 \%$ of gaps. For the remaining sequences, the 
gap character "-" was removed, since it has no meaning and was entered by the alignment algorithm. For instance, the sequence " - . - ACAGAA - . - ." becomes "ACAGAA ", while the sequences "ACA-GUA" and "ACA- -GUA" becomes "ACAGUA".

The alignment, filling, selection and segmentation procedure ended up generating 10 databases, one for each protein. Furthermore, based on the hypothesis that identical samples could be used in several researches and that, moreover, duplicate samples do not add value to the learning of a ML classifier, identical sequences of the same coding protein were eliminated. After that, the final distribution of the bases can be seen in Table 1.

\section{Normalization}

The normalization step consists of analyzing the nucleotides of the sequences, standardizing nucleotides without biological meaning, probably caused by sequencing errors. Therefore, in normalization, nucleotides that are not defined in the IUPAC nucleotide code are replaced by the pattern character I that represents indeterminacy.

\section{Tokenization}

Tokenization consists of segmenting each sequence into smaller subsequences, obtaining an ordered list of these subsegments. In our experiments, codons are the sequence substructure used for tokenization. Codons consist of nucleotide triplets that can be transcribed to amino acids [33]. Then, in the tokenization step, the amino acids of each protein sequence are obtained.

\section{Amino acid co-occurrence matrices}

Co-occurrence matrices have been used to collect statistics from varied data, especially image and text data [34-36]. In medical image analysis, co-occurrence matrices are used to measure image textures [37]. In the field of Natural Language Processing (NLP), co-occurrences can provide clues to semantic relationships between words in a body of text [38]. The application of co-occurrence matrices also expands into the field of bioinformatics, for example, in protein sequences, evidence of important functional

Table 1 Generated database distributions

\begin{tabular}{|c|c|c|c|c|c|}
\hline \multirow[t]{2}{*}{ Protein } & \multicolumn{5}{|c|}{ Samples } \\
\hline & Total & Duplicates & Remaining & Classic & Severe \\
\hline C & 298 & 192 & 106 & 71 & 35 \\
\hline M & 288 & 155 & 133 & 91 & 42 \\
\hline$E$ & 394 & 123 & 271 & 190 & 81 \\
\hline NS1 & 275 & 118 & 157 & 110 & 47 \\
\hline NS2A & 270 & 132 & 138 & 104 & 34 \\
\hline NS2B & 270 & 167 & 103 & 70 & 33 \\
\hline NS3 & 270 & 88 & 182 & 132 & 50 \\
\hline NS4A & 270 & 145 & 125 & 85 & 40 \\
\hline NS4B & 270 & 144 & 126 & 89 & 37 \\
\hline NS5 & 270 & 60 & 210 & 148 & 62 \\
\hline
\end{tabular}


relationships for protein biological processes can be found when identical patterns of amino acid co-occurrence are present in different regions [39, 40].

A amino acid co-occurrence is the occurrence of two amino acid in a protein segment. Let $P$ be a sequence of amino acid and $S$ a segment of $P$, the co-occurrence matrix $X$ can be obtained by the formula: $X_{i j}=\sum_{S} K_{i j}$, where,

$$
K_{i j}=\left\{\begin{array}{l}
1, \text { if } i, j \in S \\
0, \text { otherwise }
\end{array}\right.
$$

and $X_{i j}$ denotes the number of times amino acid $j$ was in the same segment as amino acid $i$. Thus, $X_{i, j}$ is proportional to the joint probability $P(i, j)$, which represents the probability of occurrence of the terms $i$ and $j$ in the same segment.

The segment, or context window, reflects on the type of information provided by the matrices, for example, large segments reflect the coverage of large areas of the genome, generating co-occurrences between distant amino acid and reflecting on the ability of the co-occurrence matrices to capture long-distance correlations. Similarly, small segments define a search for closer patterns within a small region.

In order for the co-occurrence matrices of each sample of the same coding region to have identical dimensions, it was necessary to create a global dictionary containing all amino acids present in the samples. With possession of the global dictionary, it was possible to generate a template co-occurrence matrix that integrates all its co-occurrences. For example, let the samples be $A_{1}=\{[C A U][I C G][G G C]\}, A_{2}=\{[C A U][G C G][U G U]\}$ and $A_{3}=\{[G A U][G C G][A I C]\}$ it is possible to get the global amino acid dictionary $d=$ $\{C A U, I C G, G G C, G C G, U G U, G A U, A I C\}$ which allows us to generate the template cooccurrence matrix present in Fig. 3. The fact that co-occurrences are interchangeable generates a symmetrical co-occurrence matrix.

\section{Co-occurrence matrix resizing and vectorizing}

Based on the symmetry of the co-occurrence matrices, the first scaling step is to extract only elements of the upper triangular matrix. The generated co-occurrence matrices have dimensions $\mathbb{R}^{d \times d}$, where $d$ is the size of the amino acid dictionary. The fact that the matrices are symmetric and interchangeable allows the resizing of the upper triangular matrix into a vector of dimension $\mathbb{R}^{d(d+1) / 2 \times 1}$. Finally, through these vectors it is possible to build a tabular database, where each column of the base represents a co-occurrence between pairs of amino acids.

\begin{tabular}{|c|c|c|c|c|c|c|c|}
\cline { 2 - 7 } \multicolumn{1}{c|}{} & CAU & ICG & GGC & GCG & UGU & GAU & AIC \\
\hline CAU & CAU CAU & CAU ICG & CAU GGC & CAU GCG & CAU UGU & CAU GAU & CAU AIC \\
\hline ICG & CAU ICG & ICG ICG & ICG GGC & ICG GCG & ICG UGU & ICG GAU & ICG AIC \\
\hline GGC & CAU GGC & ICG GGC & GGC GGC & GGC GCG & GGC UGU & GGC GAU & GGC AIC \\
\hline GCG & CAU GCG & ICG GCG & GGC GCG & GCG GCG & GCG UGU & GCG GAU & GCG AIC \\
\hline UGU & CAU UGU & ICG UGU & GGC UGU & GCG UGU & UGU UGU & UGU GAU & UGU AIC \\
\hline GAU & CAU GAU & ICG GAU & GGC GAU & GCG GAU & UGU GAU & GAU GAU & GAU AIC \\
\hline AIC & CAU AIC & ICG AIC & GGC AIC & GCG AIC & UGU AIC & GAU AIC & AIC AIC \\
\hline
\end{tabular}

Fig. 3 Co-occurrence matrix. A template matrix for samples $A_{1}, A_{2}$ and $A_{3}$ 


\section{Feature selection}

In order to achieve maximum classifier performance by reducing problem complexity and eventually an overfitting, we eliminate co-occurrences that carry little or no information. For this, we use the Mutual Information (MI) algorithm that measures the dependence between two variables by calculating entropy using the k-nearest neighbors. In this context, two variables can be considered independent if, and only if, the MI coefficient between them is zero. In contrast, the greater the dependence between two variables, the greater their mutual information value [41, 42]. Therefore, mutual information values between co-occurrences and clinical picture were calculated for each protein base. Finally, the 50 co-occurrences that presented the greatest mutual information related to the clinical picture of dengue were selected for each database.

\section{Random-forest}

The scarcity of publicly available samples with the clinical outcomes makes complex classification algorithms like CNN and LSTM have great difficulties in learning patterns in our data, considering the large amount of samples that these algorithms require for parameter optimization. Therefore, we chose to use the Random-Forest (RF) classifier for our experiments. Overall, RF classifiers are significantly less complex than deep machine learning methods, yet they are still widely used in the field of bioinformatics [43-47]. RF (Fig. 4) can be defined as models that consist of structured collections of $\left\{h\left(x, \Theta_{k}\right), k=1, \ldots\right\}$ decision trees , where $\Theta_{k}$ are independent and identically distributed variables and $x$ is an input vector. After generating the trees, RF selects the most popular class among the trees for input $x$ [48].

The RFs are part of a set of methods called ensembles, which are nothing more than combinations of several models to obtain a single result, making the ensembles more

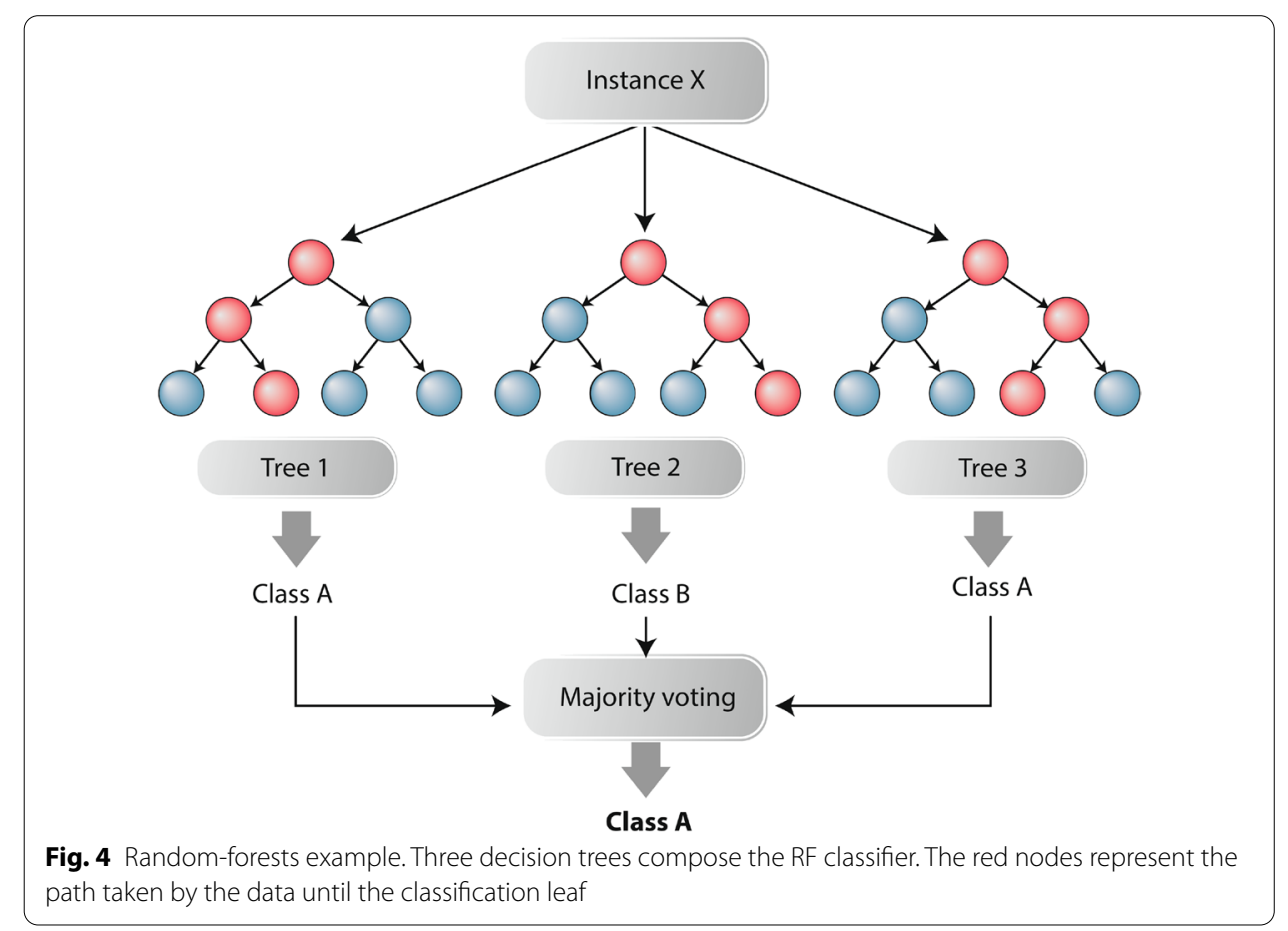


robust when compared to simpler algorithms such as trees decision or $\mathrm{kNN}[49,50]$. The basic structure of RF have as their basic unit binary decision trees (binary estimators) that employ recursive data partitioning.

To build each decision tree, the algorithm randomly selects variables from the training data and, from these, selects the most informative one to be the initial node (root node) that will have the first condition verified, giving rise to two child nodes that will initiate branches to the left and right of the root node. The node generation process is repeated throughout the tree, determining rules that define the data flow through the tree's branches and establish its decision making $[43,51]$. All these processes are repeated in the generation of the next trees. Finally, the RF defines the predicted class based on the class vote of the generated $n$-trees, where, the most predicted class in all the trees will be the final class of the RF [43].

\section{Model explainability with SHAP Values}

Many machine learning algorithms are considered functional black boxes because, given their complexity, it is almost impossible to understand their internal processes. However, in bioinformatics it is essential that there is a human domain over the classifier's decisions. Given this issue, several explainability methods have been proposed to explore the decisions made by ML models by evaluating the influence of input variables on the prediction results [52-56].

We can also mention other explanation techniques used in biological sequence classification problems through Deep Learning (DL) models [57-59], where the classifier is a Convolution Neural Network (CNN). Therefore, in these works it is assumed that the explanations are linked to the significant values of the CNN filters and the positions in which these values occur, then these values are backtracked to the input sequence and the relevant patterns are collected. As they are DL-based models, they need large amounts of data to be trained and explained, and unfortunately, our small amount of samples makes it impossible to use DL-based methods. Therefore, given the limitations imposed by the amount of samples, we chose the Random Forest classifier and used the SHAP values method with its specific explainer for tree-based models.

Explainability methods are divided into two classes: global methods that explain model results for all data inputs; and local methods that explain an individual input. Our interest in model explanation is to be able to understand what happens in the classification of severe dengue, making it possible to identify significant amino acids co-occurrences for classifier assign a sample to the severe dengue class. Therefore, in explaining the model we want to encode its learned patterns and decision-making into information explainable in human terms.

Therefore, we decided to use in our experiments the SHAP Values [54] method that performs a local explanation under the trained model and the instance of interest, making it possible to independently interpret classical dengue samples and severe dengue samples. The basic concept of SHAP Values is to ensure that two models $f$ and $g$ have approximate results for each instance. For this to occur, the condition $g\left(x^{\prime}\right)-f\left(h_{x}\left(x^{\prime}\right)\right)$ ), where $f$ is the original predictive model, $g$ is the interpreter model, and $x^{\prime}$ is a simplification of the original instance $x$ that can be mapped to the original instance from a function $h$, such that $x=h_{x}\left(x^{\prime}\right)$. For a more detailed understanding, SHAP Values unifies the 
importance of variables through a conditional expected value function of the $f$ model, such that, $f_{x}\left(z^{\prime}\right)=f\left(h_{x}\left(x^{\prime}\right)\right)=E\left(f(x) \mid x_{S}\right)$, where $S$ is the non-null subset of $x^{\prime}$. Finally, the general equation of the method explanation model takes the form of the conditional expectation function $f\left(h_{x}\left(x^{\prime}\right)\right)=E\left(f(x) \mid x_{S}\right)$ [54].

\section{TreeExplainers}

TreeExplainer is a specific method for local explanations of tree-based models, providing fast and accurate results by calculating the SHAP values for each leaf of a tree. The algorithms estimate $f\left(h_{x}\left(x^{\prime}\right)\right)=E\left(f(x) \mid x_{S}\right)$ recursively following the decision path for an input instance $x$ in a tree. The complete methodology, as well as the algorithms that define the TreeExplainer, can be found at [60].

\section{SHAP Values explanations results}

Machine learning models internally perform multiple mathematical operations to obtain results. For example, to perform predictions, classifiers generate real values which in turn will be associated with labels. As described earlier, SHAP Values performs variable explanation from the conditional expectation function.

From there, the method assigns positive and negative impacts to the input instance variables so that the expected value of the interpreter $E\left(f(x) \mid x_{S}\right)$ is equal to the output value of the original model $f$. Thus, the magnitude of the impact reflects the influence of the variable in the classification of the sample, such that positive impacts increase the probability of correct classification of the sample, while negative impacts have the opposite effect, suggesting that variables with positive impacts have a greater capacity to characterize the sample class [61]. Therefore, for each sample, the SHAP Values method generates a table that associates a classification impact value with the features in the sample.

To facilitate viewing the patterns provided by SHAP, we chose to generate a global explanation from multiple local explanations. For this purpose, after obtaining all the tables, the positive impact score of each co-occurrence is calculated, which consists of the number of times each co-occurrence had a positive impact divided by the number of times the co-occurrence appeared. Then, the average impact value of each of them is calculated. After that, each co-occurrence is ranked in descending order by the two metrics. Finally, we selected the resulting co-occurrences located in the first $20 \%$ ranking positions and the final $20 \%$. That is, the $20 \%$ with the highest positive impact and the highest positive impact score and the $20 \%$ with the lowest positive impact and lowest positive impact score.

\section{Experiments and results}

Five stratified cross-validations were performed to observe the classifier's response on different training and test sets. In view of the evident unbalance of classes in the bases presented in the Table 1, the PR-AUC metric (Area Under the Precision-Recall Curve) [62] was chosen to evaluate the model, in addition to the metrics: ROC-AUC metric (Area Under the ROC Curve), precision, recall and balanced F1-score. Precision, recall, and F1-score balanced metrics compensate for class imbalance by calculating a weighted average across correctly classified instances, while ROC-AUC is 
Table 2 Average of the metrics obtained in fivefolds cross validation with $95 \%$ of confidence interval using Student's t-distribution

\begin{tabular}{llllll}
\hline Protein & $\begin{array}{l}\text { PR-AUC } \\
\text { Mean } \pm \boldsymbol{E}\end{array}$ & $\begin{array}{l}\text { ROC-AUC } \\
\text { Mean } \pm \boldsymbol{E}\end{array}$ & $\begin{array}{l}\mathbf{P} \\
\text { Mean } \pm \boldsymbol{E}\end{array}$ & $\begin{array}{l}\text { R } \\
\text { Mean } \pm \boldsymbol{E}\end{array}$ & $\begin{array}{l}\text { M 1 } \\
\end{array}$ \\
\hline C & $0.66 \pm 0.06$ & $0.77 \pm 0.04$ & $0.74 \pm 0.03$ & $0.70 \pm 0.03$ & $0.70 \pm 0.03$ \\
M & $0.57 \pm 0.06$ & $0.73 \pm 0.04$ & $0.71 \pm 0.03$ & $0.70 \pm 0.03$ & $0.70 \pm 0.03$ \\
E & $\mathbf{0 . 6 7} \pm \mathbf{0 . 0 4}$ & $\mathbf{0 . 8 5} \pm \mathbf{0 . 0 2}$ & $\mathbf{0 . 8 0} \pm \mathbf{0 . 0 2}$ & $\mathbf{0 . 7 9} \pm \mathbf{0 . 0 2}$ & $\mathbf{0 . 7 9} \pm \mathbf{0 . 0 2}$ \\
NS1 & $0.62 \pm 0.06$ & $0.74 \pm 0.04$ & $0.72 \pm 0.02$ & $0.69 \pm 0.03$ & $0.70 \pm 0.03$ \\
NS2A & $0.52 \pm 0.06$ & $0.73 \pm 0.04$ & $0.74 \pm 0.02$ & $0.70 \pm 0.03$ & $0.71 \pm 0.03$ \\
NS2B & $0.57 \pm 0.05$ & $0.74 \pm 0.03$ & $0.73 \pm 0.03$ & $0.70 \pm 0.03$ & $0.70 \pm 0.03$ \\
NS3 & $0.58 \pm 0.05$ & $0.75 \pm 0.04$ & $0.73 \pm 0.02$ & $0.71 \pm 0.02$ & $0.71 \pm 0.02$ \\
NS4A & $0.55 \pm 0.06$ & $0.71 \pm 0.05$ & $0.72 \pm 0.04$ & $0.69 \pm 0.04$ & $0.69 \pm 0.04$ \\
NS4B & $0.58 \pm 0.05$ & $0.74 \pm 0.04$ & $0.70 \pm 0.03$ & $0.67 \pm 0.03$ & $0.68 \pm 0.03$ \\
NS5 & $0.52 \pm 0.05$ & $0.75 \pm 0.03$ & $0.73 \pm 0.03$ & $0.71 \pm 0.03$ & $0.71 \pm 0.03$ \\
\hline
\end{tabular}

Highlight the best results obtained through bold text

The P, R and F1 columns represent the Precision, Recall and F1-Score metrics, respectively

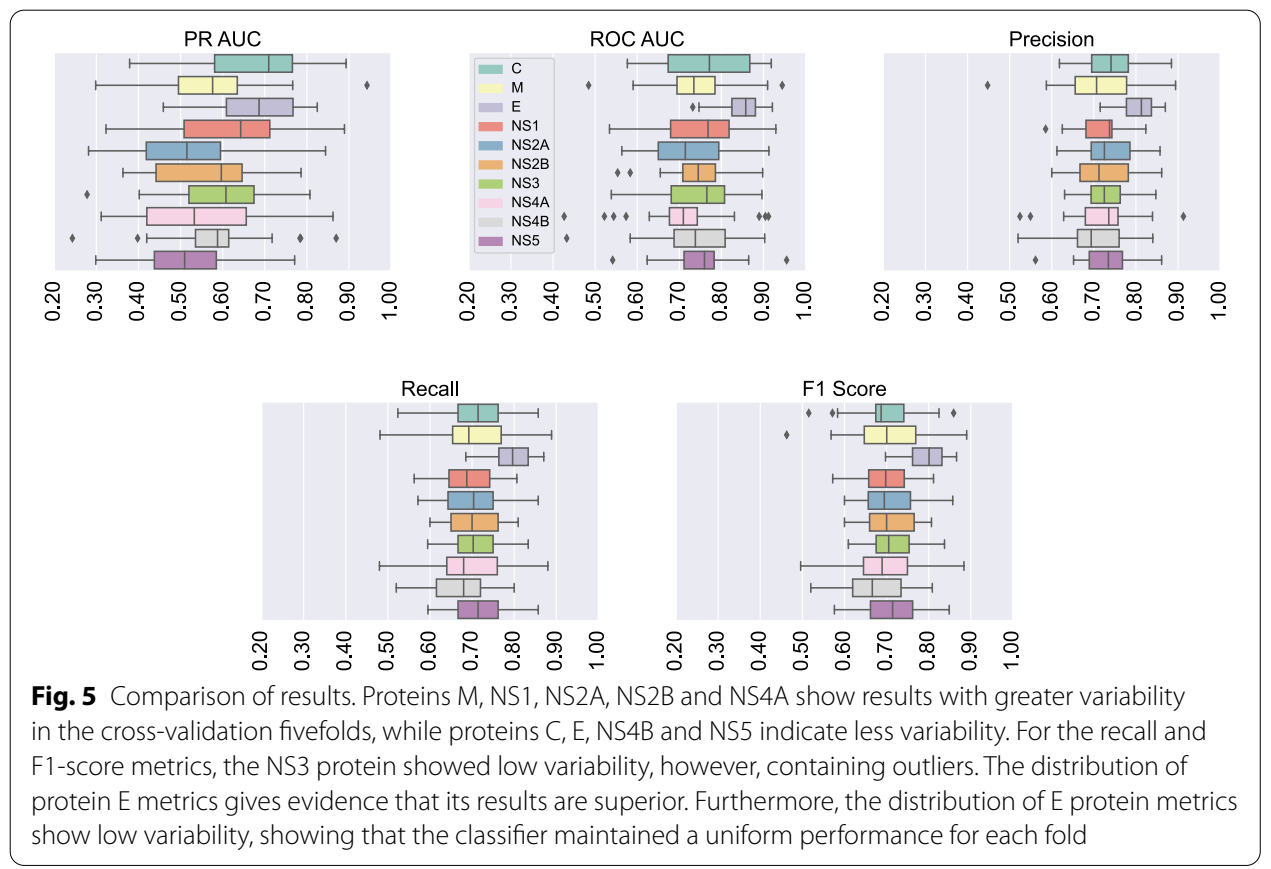

more optimistic than PR-AUC for unbalanced datasets. The mean of the metrics, as well as their confidence intervals for all proteins can be seen in Table 2. Also, we perform exploratory analyzes to observe the classifier performance in each database. To visually compare the results obtained for each database, we used box-plots (Fig. 5) to verify the empirical distribution of the metrics.

It is possible to observe in the box-plots in Fig. 5 that for the fivefolds of validation, the results of each metric for proteins M, NS1 ,NS2A and NS4A have a high variance when compared to the other proteins. On the other hand, the box-plots of protein $\mathrm{E}$ have low variance in Precision, Recall and F1-score metrics, indicating that for each 
fold the results obtained are more constant than in the other proteins, which suggests a greater capacity for generalization by the classifier when it uses protein $\mathrm{E}$ data.

Furthermore, the box-plots of the Precision, Recall and F1-score metrics in Fig. 5 show a possible difference between the results obtained for each protein. Therefore, to statistically test the hypothesis that the mean results are different for each protein, we used the one-way analysis of variance (ANOVA) model, which compares sample means through the Fisher-Snedecor F distribution [63, 64]. The ANOVA test hypotheses are: the null hypothesis $H_{0}$, where the sample means are equal, and the alternative hypothesis $H_{1}$, where at least one of the averages is different from the others.

The data used in the ANOVA test must meet the assumption of homogeneity of variances, verified by the Levene test [65], as well as the model's residuals must be normally distributed, verified by the Shapiro-Wilk test [66]. The null $\left(H_{0}\right)$ and alternative $\left(H_{1}\right)$ hypotheses for Levene's test are: the groups variances are homogeneous and the groups variances are not homogeneous, respectively. For the Shapiro-Wilk test the hypotheses are: $H_{0}$ data is normally distributed and $H_{1}$ : data is not normally distributed. All null hypotheses are accepted if, and only if, the p-value of the test is greater than a significance level of $\epsilon$. The Table 3 presents the results of the ANOVA tests for each metric, as well as the tests of their assumptions.

After obtaining the confirmations of the ANOVA test, we applied the Tukey test to verify the difference between the means of the metrics for each protein. The null hypothesis for Tukey's test assumes that there is no statistically significant difference between the means of two samples, while the alternative hypothesis assumes the opposite. Protein pairs with statistically distinct means of metrics can be seen in Fig. 6. As we can see, for all metrics, protein $\mathrm{E}$ presents statistically different averages at least one protein in Tukey pair comparison.

\section{Explanations}

After being trained, the classifiers were interpreted using the SHAP Values method through the TreeExplainer algorithm. The SHAP Values method generates individual explanations for each data sample. For our explanations we use force plots, which in turn show the impact of sample variables on the prediction [61]. Then, from the force plots we can extract the impact of each co-occurrence on the probability of classification of

Table 3 For a significance level of $\epsilon=0.05$ the Levene and Shapiro-Wilk tests show evidence that the metrics have homogeneous variances and that the residuals of the ANOVA model are normally distributed

\begin{tabular}{llll}
\hline & Leven's test $\boldsymbol{p}$ value & ANOVA $\boldsymbol{p}$ value & $\begin{array}{l}\text { Shapiro- } \\
\text { Wilk } \boldsymbol{p} \\
\text { value }\end{array}$ \\
\hline PR-AUC & & $4 \times 10^{-5}$ & 0.83 \\
ROC-AUC & 0.83 & $6 \times 10^{-5}$ & 0.09 \\
Precision & 0.10 & $4 \times 10^{-4}$ & 0.43 \\
Recall & 0.07 & $2 \times 10^{-5}$ & 0.53 \\
F1-score & 0.11 & $2 \times 10^{-5}$ & 0.63 \\
\hline
\end{tabular}

Finally, the null hypothesis of the ANOVA test is rejected, indicating that at least one of the metric means is different from the others 


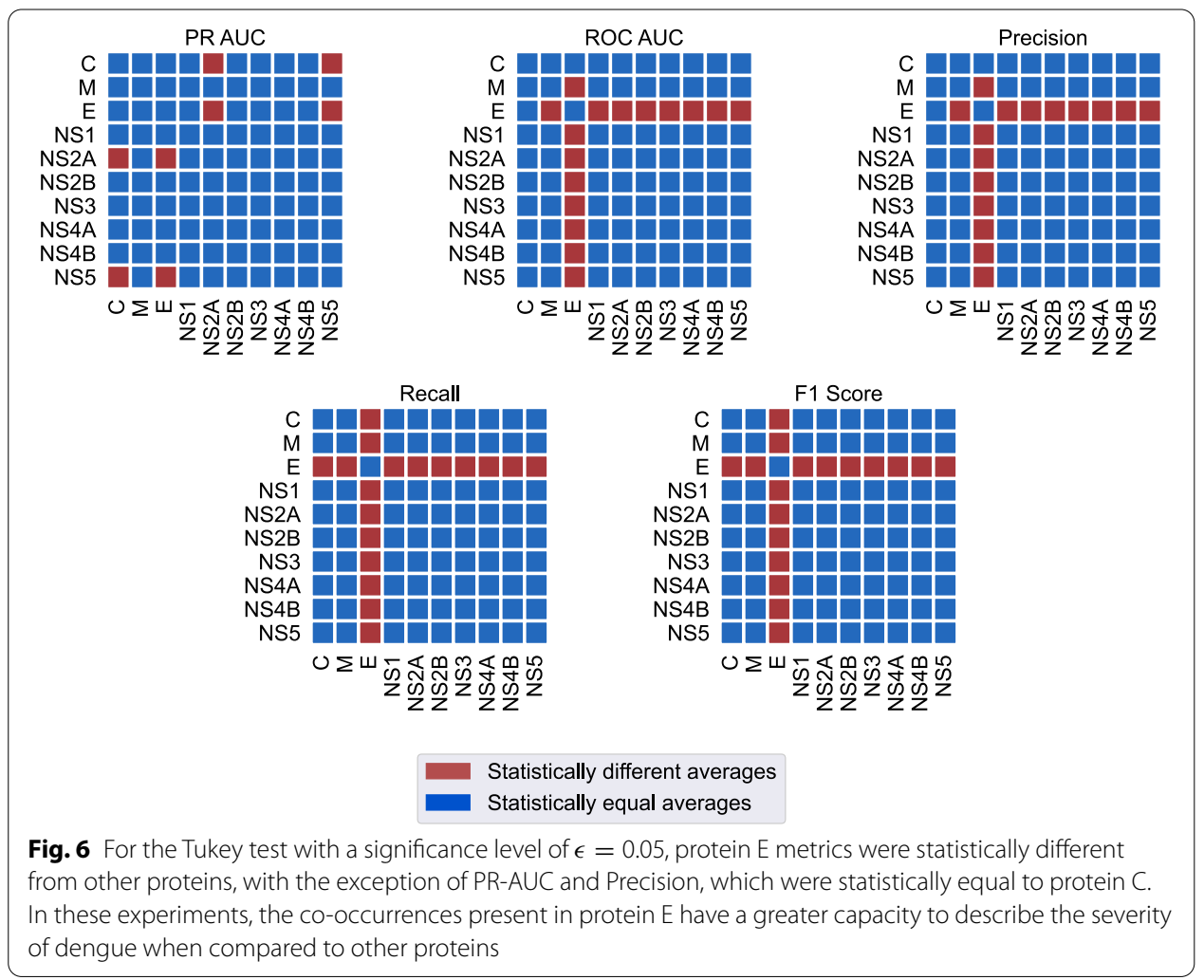

severe dengue. Therefore, the first step of our explanations is to rank the co-occurrences that increase the probability of severe dengue, so that, finally, we can visualize the distribution of these co-occurrences and their behavior in samples of classic dengue.

Of the 50 co-occurrences selected by the MI algorithm, the explanation graphs will be $20 \%$ of the most relevant co-occurrences in the classification of severe dengue and the $20 \%$ less relevant. Finally, the co-occurrence values will be compared with classic dengue samples. As stated earlier, explanations generate positive and negative impacts. Cooccurrences do not have a constant impact behavior for each sample, that is, the same co-occurrence may have positive impacts in certain samples and negative impacts in severe dengue samples.

\section{E protein explanations plots}

Protein E explanations reveal distinct characteristics between co-occurrences of significant amino acids for severe dengue compared to classic dengue. In general, as we can see in Fig. 7, the co-occurrence distributions are mostly distinct for classic and severe dengue. Examining the Fig. 7 we can observe differences in the behavior of the empirical distributions of amino acids significant for severe dengue compared with their behavior in classic dengue. These differences are more evident for the co-occurrence between the amino acids Serine and Tryptophan (encoded by UCA and UGG, respectively) which is positively significant in $96 \%$ of severe dengue samples. In this we can observe that the value distribution of this co-occurrence tends to have higher concentrations, close to 10 , while for severe dengue this figure rises to 20 . 


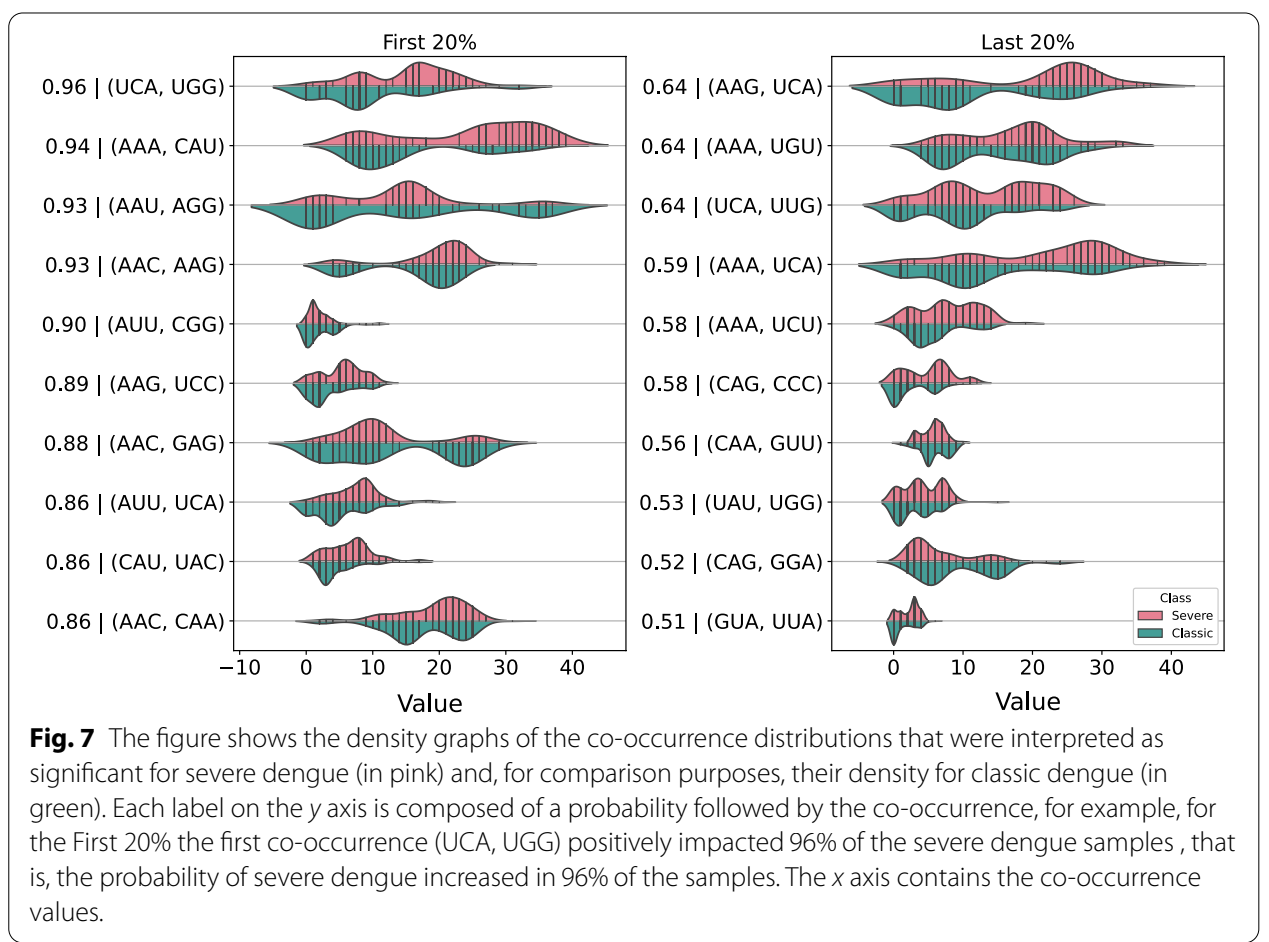

We can observe that for all cases the empirical distributions of significant co-occurrences for severe dengue are not graphically identical to those for classic dengue, although they are close in some cases. Again, it is important to emphasize that the co-occurrences present in Fig. 7 are ranked according to their importance in the classification of severe dengue in the samples. For example, the first co-occurrence (UCA, UGG) was significant for classification of $96 \%$ of severe dengue samples, while the last co-occurrence (AAG, CGC) was significant for classification of only $35 \%$ of severe dengue samples.

\section{Co-occurrences importance by $E$ protein regions}

Dengue E protein can be divided into four major regions, namely: Domain 1, Domain 2, Transmembrane 1 and Transmembrane 2. Each of the four dengue serotypes have specific RNA positions that mark the beginning and end of these regions [67-72]. To improve the visualization, after analyzing the behavior of the co-occurrences for samples of each serotype, the co-occurrence values by region for samples of each serotype are grouped through the mean, as can be seen in Table 4 .

The Domain 1 region of dengue $\mathrm{E}$ protein has the highest mean concentration of significant co-occurrences for the classification of severe dengue. With the exception of the co-occurrence (GUA, UAA) which is on average more present in Domain 2, all the others are more frequent in Domain 1, as we can see in Table 4. This is an indication that domain 1 may be directly related to the probability of dengue fever in the clinical outcome. However, more in-depth experiments are needed to confirm this evidence. 
Table 4 Domain 1 has on average more significant co-occurrences for severe dengue

\begin{tabular}{|c|c|c|c|c|}
\hline Co-occurrence & Domain 1 & Domain 2 & Transmembrane 1 & $\begin{array}{l}\text { Transmembrane } \\
2\end{array}$ \\
\hline (UCA, UGG) & 4.33 & 1.81 & 0.79 & 0.06 \\
\hline$(\mathrm{AAA}, \mathrm{CAU})$ & 10.30 & 3.07 & 0.03 & 0.42 \\
\hline$(A A U, A G G)$ & 8.30 & 0.48 & 1.28 & 0.22 \\
\hline (AAC, AAG) & 8.98 & 3.45 & 0.00 & 0.00 \\
\hline$(A \cup U, C G G)$ & 1.40 & 0.06 & 0.34 & 0.00 \\
\hline (AAG, UCC) & 1.22 & 1.06 & 0.03 & 0.00 \\
\hline (AAC, GAG) & 6.12 & 4.06 & 0.00 & 0.00 \\
\hline$(A \cup U, \cup C A)$ & 2.51 & 1.40 & 0.66 & 0.03 \\
\hline$(C A \cup, \cup A C)$ & 2.78 & 1.52 & 0.00 & 0.00 \\
\hline$(A A C, C A A)$ & 10.19 & 3.35 & 0.00 & 0.00 \\
\hline$(A A G, U C A)$ & 5.51 & 3.51 & 0.04 & 0.00 \\
\hline (AAA, UGU) & 8.94 & 2.12 & 0.00 & 0.00 \\
\hline (UCA, UUG) & 3.89 & 1.64 & 0.46 & 0.12 \\
\hline (AAA, UCA) & 5.69 & 4.37 & 0.08 & 0.81 \\
\hline (AAA, UCU) & 2.75 & 1.15 & 0.12 & 0.06 \\
\hline (CAG, CCC) & 1.72 & 0.32 & 0.31 & 0.00 \\
\hline (CAA, GUU) & 3.16 & 0.79 & 0.00 & 0.00 \\
\hline (UAU, UGG) & 0.85 & 0.07 & 0.12 & 0.27 \\
\hline (CAG, GGA) & 9.05 & 0.69 & 0.01 & 0.00 \\
\hline (GUA, UUA) & 0.61 & 0.76 & 0.12 & 0.00 \\
\hline
\end{tabular}

For E protein regions, highlight, through the bold text, the highest average value of co-occurrences for the significant amino acid pairs

\section{Discussion}

In this article, we present a method capable of representing and classifying severe dengue according to the protein coding sequence of the virus. Furthermore, the method is focused on improving the extraction of significant patterns for the classifier. The procedure is based on the segmentation of dengue viral RNA in each of the ten protein coding sequences, transforming these protein segments into matrices of co-occurrence of amino acids within a context window that will be classified by a RF.

The significant co-occurrences for severe dengue class were obtained through the SHAP Values explanation model, which employs a range of strategies to select variables that have greater weight in the classifier's decision making, that is, co-occurrences that increase the probability of severe dengue. An important piece of information is that the context window is not automatically generated, this allows one to adjust the range of cooccurrences, allowing one to choose between performing local analyses, represented by patterns of co-occurrences conserved within the genome, or analyzes in large segments, allowing for co-occurrences between distant amino acids to be captured, increasing the chance of collecting long-distance correlations between amino acids.

Another important point to highlight is that by applying a classifier with few hyper parameters for adjustment, we reduce the need to use large databases for classification. Therefore, our method is able to perform on small databases, however, this does not mean that additional strategies are excluded, in our problem, for example, it was necessary to binarize labels to reduce the negative effects of high unbalance of our base. One of the advantages of using an RF as a classifier is that, because it is a rule-based classifier, 
the significant patterns for classification obtained by the SHAP method tend to be more concrete, since this classifier does not employ transformations in the input data, as with the deep models CNN and LSTM [73].

Finally, we emphasize that the focus of our approach is the exploratory analysis of the RNA sequences that produced a clinical outcome known as dengue severe, showing amino acid patterns that were related to this event. The presented methodology is flexible, as it would be possible to add metadata along with the co-occurrence vectors, such as mass, volume, polarity and charge of the protein segment. There are no limitations on the use of our method for classifying and interpreting other biological sequences.

\section{Conclusion}

In this work, we described an ML method capable of identifying amino acid cooccurence patterns associated with severe dengue cases. In our analysis, precisely the same amino acids didn't need to be found in all cases, but a signature of them. The biological basis of these results needs further evaluation, and other multifactorial aspects linked to dengue severe cases like secondary infection and host immunogenetics must not be ruled out. On the other hand, the method may be used as an interesting approach to identify patterns that may not be easily identified using other techniques.Moreover, the statistical analysis results do not support that the presented results occurred only by chance. Notwithstanding, the paucity of genomes with available outcome metadata may limit the robustness of some of the observed associations. Furthermore, we believe that the method described here may also be helpful for other studies with different viral agents.

\section{Supplementary Information}

The online version contains supplementary material available at https://doi.org/10.1186/s12859-022-04597-y.

Additional file 1. C protein: A list of sequences belonging to Dengue virus protein $C$ in csvformat. Each row is a sample of the amino acid chain labeledaccording to dengue severity.

Additional file 2. M protein: A list of sequences belonging to Dengue virus protein $M$ in csvformat. Each row is a sample of the amino acid chain labeledaccording to dengue severity.

Additional file 3. E protein: A list of sequences belonging to Dengue virus protein $\mathrm{E}$ in csvformat. Each row is a sample of the amino acid chain labeledaccording to dengue severity.

Additional file 4. NS1 protein: A list of sequences belonging to Dengue virus protein NS1 in csvformat. Each row is a sample of the amino acid chain labeledaccording to dengue severity.

Additional file 5. NS2A protein: A list of sequences belonging to Dengue virus protein NS2A in csvformat. Each row is a sample of the amino acid chain labeledaccording to dengue severity.

Additional file 6. NS2B protein: A list of sequences belonging to Dengue virus protein NS2B in csvformat. Each row is a sample of the amino acid chain labeledaccording to dengue severity.

Additional file 7. NS3 protein: A list of sequences belonging to Dengue virus protein NS3 in csvformat. Each row is a sample of the amino acid chain labeledaccording to dengue severity

Additional file 8. NS4A protein:A list of sequences belonging to Dengue virus protein NS4A in csvformat. Each row is a sample of the amino acid chain labeledaccording to dengue severity.

Additional file 9. NS4B protein: A list of sequences belonging to Dengue virus protein NS4B in csvformat. Each row is a sample of the amino acid chain labeledaccording to dengue severity.

Additional file 10. NS5 protein: A list of sequences belonging to Dengue virus protein NS5 in csvformat. Each row is a sample of the amino acid chain labeledaccording to dengue severity. 
of Amazonia Ltda, under the terms of Federal Law n ${ }^{\circ}$ 8.387/1991, through agreement $n^{\circ}$ 003/2019, signed with ICOMP/ UFAM. This research, carried out within the scope of the Samsung-UFAM Project for Education and Research (SUPER), according to Article 48 of Decree $n^{\circ}$ 6.008/2006(SUFRAMA), was funded by Samsung Electronics of Amazonia Ltda, under the terms of Federal Law $n^{\circ}$ 8.387/1991, through agreement 001/2020, signed with Federal University of Amazonas and FAEPI, Brazil. This research, according to Article 48 of Decree $n^{\circ} 6.008 / 2006$, was partially funded by Samsung Electronics of Amazonia Ltda, under the terms of Federal Law n ${ }^{\circ} 8.387 / 1991$, through agreement $n^{\circ} 003 / 2019$, signed with ICOMP/UFAM

\section{Authors' contributions}

LS worked on Methodology and Experimental Design, Algorithm Implementation, Writing Original Draft. JC worked on Methodology and Experimental Design, Results Verification, Draft Review. JM worked on Data Acquisition and Analysis, Draft Review. FN worked on Conceptualization, Data Analysis, Draft Review. All authors read and approved the final manuscript.

\section{Funding}

Not applicable.

Availability of data and materials

The datasets generated and/or analysed during the current study are available in the Github repository, https://doi.org/ $10.5281 /$ zenodo.5885637

\section{Declarations}

\section{Ethics approval and consent to participate}

Not applicable.

\section{Consent for publication}

Not applicable.

\section{Competing interests}

The authors declare that they have no competing interests.

\section{Author details}

${ }^{1}$ Institute of Computing, Federal University of Amazonas, General Rodrigo Octavio Avenue, Manaus, Amazonas, Brazil. ${ }^{2}$ Institute of Biological Sciences, Federal University of Amazonas, General Rodrigo Octavio Avenue, Manaus, Amazonas, Brazil. 'eonidas and Maria Deane Institute, Oswaldo Cruz Foundation, Terezina Street, Manaus, Amazonas, Brazil.

Received: 21 August 2021 Accepted: 2 February 2022

Published online: 19 February 2022

\section{References}

1. Shope RE, Meegan JM. In: Evans AS, Kaslow RA, editors. Arboviruses. Boston: Springer; 1997. p. 151-183.

2. Organization WH, for Research SP, in Tropical Diseases T, of Control of Neglected Tropical Diseases WHOD, Epidemic WHO. Alert, P. Dengue: guidelines for diagnosis, treatment, prevention and control. World Health Organization; 2009. https://apps.who.int/iris/handle/10665/44188.

3. Organization WH et al. Comprehensive guideline for prevention and control of dengue and dengue haemorrhagic fever; 2011. pp. 3-7.

4. Honório NA, Silva WdC, Leite PJ, Gonçalves JM, Lounibos LP, Lourenço-de-Oliveira R. Dispersal of Aedes aegypti and Aedes albopictus (diptera: Culicidae) in an urban endemic dengue area in the State of Rio de Janeiro, Brazil. Mem Inst Oswaldo Cruz. 2003;98(2):191-8.

5. Eisen L, Moore CG. Aedes (stegomyia) aegypti in the continental united states: a vector at the cool margin of its geographic range. J Med Entomol. 2013;50(3):467-78.

6. Bhatt S, Gething PW, Brady OJ, Messina JP, Farlow AW, Moyes CL, Drake JM, Brownstein JS, Hoen AG, Sankoh O, et al. The global distribution and burden of dengue. Nature. 2013;496(7446):504-7.

7. Ong A, Sandar M, Chen MI, Sin LY. Fatal dengue hemorrhagic fever in adults during a dengue epidemic in Singapore. Int J Infect Dis. 2007;11(3):263-7.

8. Macedo GA, Gonin MLC, Pone SM, Cruz OG, Nobre FF, Brasil P. Sensitivity and specificity of the world health organization dengue classification schemes for severe dengue assessment in children in rio de janeiro. PLOS ONE. 2014;9(4):96314.

9. Brady OJ, Gething PW, Bhatt S, Messina JP, Brownstein JS, Hoen AG, Moyes CL, Farlow AW, Scott TW, Hay SI. Refining the global spatial limits of dengue virus transmission by evidence-based consensus. PLoS Negl Trop Dis. 2012;6(8):1760.

10. Kraemer MU, Sinka ME, Duda KA, Mylne AQ, Shearer FM, Barker CM, Moore CG, Carvalho RG, Coelho GE, Van Bortel W, et al. The global distribution of the arbovirus vectors Aedes aegypti and Aedes albopictus. Elife. 2015;4:08347.

11. Wilder-Smith A, Ooi E, Horstick O, Wills B. Dengue. Lancet. 2019;393(10169):350-63.

12. Sabin AB, et al. Research on dengue during World War II. Am J Trop Med Hyg. 1952;1(1):30-50. 
13. Reich NG, Shrestha S, King AA, Rohani P, Lessler J, Kalayanarooj S, Yoon I-K, Gibbons RV, Burke DS, Cummings DA. Interactions between serotypes of dengue highlight epidemiological impact of cross-immunity. J R Soc Interface. 2013;10(86):20130414.

14. Olkowski S, Forshey BM, Morrison AC, Rocha C, Vilcarromero S, Halsey ES, Kochel TJ, Scott TW, Stoddard ST. Reduced risk of disease during postsecondary dengue virus infections. J Infect Dis. 2013;208(6):1026-33.

15. Guzman MG, Halstead SB, Artsob H, Buchy P, Farrar J, Gubler DJ, Hunsperger E, Kroeger A, Margolis HS, Martínez E, et al. Dengue: a continuing global threat. Nat Rev Microbiol. 2010;8(12):7-16.

16. Mackenzie JS, Gubler DJ, Petersen LR. Emerging flaviviruses: the spread and resurgence of Japanese encephalitis, West Nile and dengue viruses. Nat Med. 2004;10(12):98-109.

17. Perera R, Kuhn RJ. Structural proteomics of dengue virus. Curr Opin Microbiol. 2008;11(4):369-77.

18. Kuhn RJ, Zhang W, Rossmann MG, Pletnev SV, Corver J, Lenches E, Jones CT, Mukhopadhyay S, Chipman PR, Strauss EG, et al. Structure of dengue virus: implications for flavivirus organization, maturation, and fusion. Cell. 2002;108(5):717-25.

19. Mackenzie JM, Khromykh AA, Jones MK, Westaway EG. Subcellular localization and some biochemical properties of the flavivirus Kunjin nonstructural proteins NS2A and NS4A. Virology. 1998;245(2):203-15.

20. Avirutnan P, Punyadee N, Noisakran S, Komoltri C, Thiemmeca S, Auethavornanan K, Jairungsri A, Kanlaya R, Tangthawornchaikul N, Puttikhunt C, et al. Vascular leakage in severe dengue virus infections: a potential role for the nonstructural viral protein NS1 and complement. J Infect Dis. 2006;193(8):1078-88.

21. Chambers TJ, MCCourt DW, Rice CM. Yellow fever virus proteins NS2A, NS213, and NS4B: identification and partial N-terminal amino acid sequence analysis. Virology. 1989;169(1):100-9.

22. Clum S, Ebner KE, Padmanabhan R. Cotranslational membrane insertion of the serine proteinase precursor NS2BNS3 (Pro) of dengue virus type 2 is required for efficient in vitro processing and is mediated through the hydrophobic regions of NS2B. J Biol Chem. 1997;272(49):30715-23.

23. Xie X, Gayen S, Kang C, Yuan Z, Shi P-Y. Membrane topology and function of dengue virus NS2A protein. J Virol. 2013;87(8):4609-22

24. Miller S, Kastner S, Krijnse-Locker J, Bühler S, Bartenschlager R. The non-structural protein 4A of dengue virus is an integral membrane protein inducing membrane alterations in a 2K-regulated manner. J Biol Chem. 2007;282(12):8873-82.

25. Tajima S, Takasaki T, Kurane I. Restoration of replication-defective dengue type 1 virus bearing mutations in the N-terminal cytoplasmic portion of NS4A by additional mutations in NS4B. Adv Virol. 2011;156(1):63-9.

26. Ray D, Shah A, Tilgner M, Guo Y, Zhao Y, Dong H, Deas TS, Zhou Y, Li H, Shi P-Y. West Nile virus 5'-cap structure is formed by sequential guanine N-7 and ribose 2'-o methylations by nonstructural protein 5. J Virol. 2006;80(17):8362-70.

27. Laurent-Rolle M, Boer EF, Lubick KJ, Wolfinbarger JB, Carmody AB, Rockx B, Liu W, Ashour J, Shupert WL, Holbrook MR, et al. The NS5 protein of the virulent West Nile virus NY99 strain is a potent antagonist of type I interferon-mediated JAK-STAT signaling. J Virol. 2010;84(7):3503-15

28. Comm I-I. Abbreviations and symbols for nucleic acids, polynucleotides, and their constituents. Biochemistry. 1970:9(20):4022-7.

29. Konstantin K, et al. Unipro UGENE: a unified bioinformaticstoolkit. Bioinformatics. 2012;28(8):1166-7.

30. Edgar RC. MUSCLE: multiple sequence alignment with high accuracy and high throughput. Nucleic Acids Res. 2004;32(5):1792-7.

31. Altschul SF, Gish W, Miller W, Myers EW, Lipman DJ. Basic local alignment search tool. J Mol Biol. 1990;215(3):403-10.

32. Thompson JD, Higgins DG, Gibson TJ. CLUSTAL W: improving the sensitivity of progressive multiple sequence alignment through sequence weighting, position-specific gap penalties and weight matrix choice. Nucleic Acids Res. 1994;22(22):4673-80.

33. Yanofsky C. Establishing the triplet nature of the genetic code. Cell. 2007;128(5):815-8.

34. Carr JR, De Miranda FP. The semivariogram in comparison to the co-occurrence matrix for classification of image texture. IEEE Trans Geosci Remote Sens. 1998:36(6):1945-52.

35. Zhang X, Cui J, Wang W, Lin C. A study for texture feature extraction of high-resolution satellite images based on a direction measure and gray level co-occurrence matrix fusion algorithm. Sensors. 2017;17(7):1474.

36. Brochier R, Guille A, Velcin J. Global vectors for node representations. In: The World Wide Web conference. 2019. p. 2587-2593.

37. Abdel-Nasser M, Moreno A, Puig D. Breast cancer detection in thermal infrared images using representation learning and texture analysis methods. Electronics. 2019;8(1):100.

38. Pennington J, Socher R, Manning CD. Glove: global vectors for word representation. In: Proceedings of the 2014 conference on empirical methods in natural language processing (EMNLP). 2014. p. 1532-1543.

39. Lee E-SA, Fung S, Sze-To H-Y, Wong AK. Confirming biological significance of co-occurrence clusters of aligned pattern clusters. In: 2013 IEEE international conference on bioinformatics and biomedicine. IEEE; 2013. p. 422-427.

40. Lee E-SA, Fung S, Sze-To H-Y, Wong AK. Discovering co-occurring patterns and their biological significance in protein families. BMC Bioinform. 2014;15(S12):2.

41. Kozachenko L, Leonenko NN. Sample estimate of the entropy of a random vector. Problemy Peredachi Informatsii. 1987;23(2):9-16.

42. Kraskov A, Stögbauer H, Grassberger P. Estimating mutual information. Phys Rev E. 2004;69(6):066138.

43. Chen X, Ishwaran H. Random forests for genomic data analysis. Genomics. 2012;99(6):323-9.

44. Ru X, Li L, Zou Q. Incorporating distance-based top-n-gram and random forest to identify electron transport proteins. J Proteome Res. 2019;18(7):2931-9.

45. Wang X, Yu B, Ma A, Chen C, Liu B, Ma Q. Protein-protein interaction sites prediction by ensemble random forests with synthetic minority oversampling technique. Bioinformatics. 2019;35(14):2395-402.

46. Wu H, Huang H, Lu W, Fu Q, Ding Y, Qiu J, Li H. Ranking near-native candidate protein structures via random forest classification. BMC Bioinform. 2019;20(25):683. 
47. Lv Z, Jin S, Ding H, Zou Q. A random forest sub-Golgi protein classifier optimized via dipeptide and amino acid composition features. Front Bioeng Biotechnol. 2019;7:215.

48. Breiman L. Random forests. Mach Learn. 2001;45(1):5-32.

49. Dietterich TG. Ensemble methods in machine learning. In: International workshop on multiple classifier systems. 2000. Springer. p. 1-15.

50. Rokach L, Schclar A, Itach E. Ensemble methods for multi-label classification. Expert Syst Appl. 2014;41 (16):7507-23.

51. Loh W-Y. Classification and regression trees. Wiley Interdiscip Rev Data Min Knowl Discov. 2011;1 (1):14-23.

52. Zafar MR, Khan NM. DLIME: a deterministic local interpretable model-agnostic explanations approach for computeraided diagnosis systems. 2019. arXiv:1906.10263.

53. Ribeiro MT, Singh S, Guestrin C. "why should i trust you?" Explaining the predictions of any classifier. In: Proceedings of the 22nd ACM SIGKDD international conference on knowledge discovery and data mining. 2016. p. 1135-1144.

54. Lundberg SM, Lee S-I. A unified approach to interpreting model predictions. In: Advances in neural information processing systems, vol. 30. 2017. p. 4765-4774.

55. Friedman JH. Greedy function approximation: a gradient boosting machine. Ann Stat. 2001;29:1189-232.

56. Goldstein A, Kapelner A, Bleich J, Pitkin E. Peeking inside the black box: visualizing statistical learning with plots of individual conditional expectation. J Comput Graph Stat. 2015;24(1):44-65.

57. Dasari CM, Bhukya R. Explainable deep neural networks for novel viral genome prediction. Appl Intell. 2021;52:1-16.

58. Amilpur S, Bhukya R. Edeepssp: explainable deep neural networks for exact splice sites prediction. J Bioinform Comput Biol. 2020;18(04):2050024.

59. Dasari CM, Bhukya R. Intersspp: investigating patterns through interpretable deep neural networks for accurate splice signal prediction. Chemom Intell Lab Syst. 2020;206:104144.

60. Lundberg SM, Erion G, Chen H, DeGrave A, Prutkin JM, Nair B, Katz R, Himmelfarb J, Bansal N, Lee S-I. From local explanations to global understanding with explainable Al for trees. Nat Mach Intell. 2020;2(1):2522-5839.

61. Lundberg SM, Nair B, Vavilala MS, Horibe M, Eisses MJ, Adams T, Liston DE, Low DK-W, Newman S-F, Kim J, et al. Explainable machine-learning predictions for the prevention of hypoxaemia during surgery. Nat Biomed Eng. 2018;2(10):749-60.

62. Davis J, Goadrich M. The relationship between precision-recall and roc curves. 2006. p. 233-240.

63. St L, Wold S, et al. Analysis of variance (ANOVA). Chemom Intell Lab Syst. 1989;6(4):259-72.

64. Girden ER. ANOVA: repeated measures, vol. 84. Thousand Oaks: Sage; 1992.

65. Levene H. Robust tests for equality of variances. Contributions to probability and statistics. Essays in honor of Harold hotelling. 1961. p. 279-292.

66. Shapiro SS, Wilk MB. An analysis of variance test for normality (complete samples). Biometrika. 1965;52(3/4):591-611.

67. Laille M, Roche C. Comparison of dengue-1 virus envelope glycoprotein gene sequences from French Polynesia. Am JTrop Med Hyg. 2004;71(4):478-84.

68. Foster JE, Bennett SN, Carrington CV, Vaughan H, McMillan WO. Phylogeography and molecular evolution of dengue 2 in the Caribbean basin, 1981-2000. Virology. 2004;324(1):48-59.

69. Li L, Lok S-M, Yu I-M, Zhang Y, Kuhn RJ, Chen J, Rossmann MG. The flavivirus precursor membrane-envelope protein complex: structure and maturation. Science. 2008;319(5871):1830-4

70. Ito M, Yamada K-I, Takasaki T, Pandey B, Nerome R, Tajima S, Morita K, Kurane I. Phylogenetic analysis of dengue viruses isolated from imported dengue patients: possible aid for determining the countries where infections occurred. J Travel Med. 2007;14(4):233-44.

71. Midgley CM, Flanagan A, Tran HB, Dejnirattisai W, Chawansuntati K, Jumnainsong A, Wongwiwat W, Duangchinda T, Mongkolsapaya J, Grimes JM, et al. Structural analysis of a dengue cross-reactive antibody complexed with envelope domain III reveals the molecular basis of cross-reactivity. J Immunol. 2012;188(10):4971-9.

72. Patil J, Cherian S, Walimbe A, Bhagat A, Vallentyne J, Kakade M, Shah P, Cecilia D. Influence of evolutionary events on the Indian subcontinent on the phylogeography of dengue type 3 and 4 viruses. Infect Genet Evol. 2012;12(8):1759-69.

73. LeCun Y, Bengio Y, Hinton G. Deep learning. Nature. 2015;521(7553):436-44.

\section{Publisher's Note}

Springer Nature remains neutral with regard to jurisdictional claims in published maps and institutional affiliations.

Ready to submit your research? Choose BMC and benefit from:

- fast, convenient online submission

- thorough peer review by experienced researchers in your field

- rapid publication on acceptance

- support for research data, including large and complex data types

- gold Open Access which fosters wider collaboration and increased citations

- maximum visibility for your research: over $100 \mathrm{M}$ website views per year

At BMC, research is always in progress.

Learn more biomedcentral.com/submissions 